\title{
Currently available medications in resource-rich settings may not be sufficient for lifelong treatment of HIV
}

\author{
James Jansson ${ }^{\mathrm{a}}$, David P. Wilson ${ }^{\mathrm{a}}$, Andrew Carr ${ }^{\mathrm{b}}$, Kathy Petoumenos ${ }^{\mathrm{a}}$, and Mark A. Boyd ${ }^{\mathrm{a}}$ \\ aThe Kirby Institute, University of New South Wales, Sydney, Australia \\ bSt Vincent's Hospital, Sydney, Australia
}

\section{Abstract}

Objective-Combination antiretroviral therapy (cART) has greatly improved the life expectancy of people living with HIV (PLHIV). Our study aims to project the life expectancy of PLHIV in a resource-rich setting in the context of the currently available antiretroviral treatments.

\begin{abstract}
Methods-Patient antiretroviral treatment data were sourced from an observational cohort of 3,434 predominantly male (94.2\%) PLHIV in Australia over the period 1997-2010. These data were analysed in a computer simulation model to calculate the distribution of time until exhaustion of all treatment options and expected effect on mortality. Standardised mortality ratios were used to simulate expected survival before and after treatment exhaustion.
\end{abstract}

\begin{abstract}
Results-We estimated that the median time until exhaustion of currently available treatment options is 45.5 years (IQR 34.0-61.0 years). However, 10\% of PLHIV are expected to exhaust all currently available cART options after just 25.6 years. PLHIV who start currently available cART regimens at age 20 years are expected to live to a median age of 67.4 (IQR 53.2-77.7) years. This is a substantial improvement on no cART (27.7 [IQR 23.8-32.0] years) but is still substantially less than the median general population mortality age (82.2 [IQR 74.0-87.8] years). The life expectancy gap between PLHIV and the general population is greatest for those infected at younger ages.
\end{abstract}

Conclusions-As treatment options are exhausted, a substantial difference in life expectancy between PLHIV and the general population could be expected even in resource-rich settings, particularly for people who acquire HIV at a younger age or who are currently highly treatment experienced.

\section{Keywords}

antiretroviral therapy; HIV; life expectancy; survival

\section{Introduction}

Since the introduction of triple combination antiretroviral therapy (cART) in the mid-1990 s there has been a remarkable turnaround in the health and wellbeing of people living with HIV (PLHIV) in settings which facilitate high access to available cART regimens. Recently, numerous studies have projected expected life expectancies of PLHIV and have concluded 
that they may approach [1,2], and in some cases even equal that of the general HIVuninfected population [3-5]. These projections rely upon the observed mortality rates in various cohort studies. The major limitation of these studies is that the relevant observations have only been gathered since the beginning of the era of combination ART in the mid-1990 $\mathrm{s}$. While the results show positive outcomes for those using cART, these results are based on short-term follow-up and they do not take into account the availability of active drugs and classes to support long-term health and HIV control over the course of decades.

The HIV armamentarium now contains an impressive number (26 as of May 2012 [6]) and range of individual agents within 6 antiretroviral drug classes. Agents in the clinical development pipeline within existing classes may have limited impact upon life expectancy if within-class cross-resistance exists between the new agents and those already available. There is currently only one agent in development in a new antiretroviral drug class [6]; the CD4 attachment inhibitor, BMS-663068. Its current status (in stage 2 clinical trial) means it cannot yet be relied upon as a future treatment for use in combination therapy to prevent HIV disease progression. This potential disconnect between life expectancy as modelled in the last few years based on short-term mortality and its support through multiple sequential fully suppressive cART regimens may become more important as prescribers move towards earlier antiretroviral initiation. Most guidelines in high-income countries are moving towards recommendations of initiation of cART on the basis of numerous studies that suggest earlier intervention is associated with less morbidity [7], lower mortality [4,5], and a public health benefit of reduced individual infectiousness [8].

In this study, we approached the question of life expectancy in PLHIV in the context of the capacity of current cART to offer long-term HIV control.

\section{Methods}

We used an agent-based computer model, which simulates disease progression, treatment progression and mortality to determine the time until treatment option exhaustion and its impact on mortality. Data for the model were sourced from an observational cohort containing data on 3,434 predominantly male $(94.2 \%)$ patients and their use of cART in a resource-rich setting over the period 1997-2010 (Australian HIV Observational Database, AHOD [9]) which provided individual-level line data on changes in antiretroviral regimen. The data were analysed to determine when individuals receive new drugs that belong to a class of drug to which they are naive. Death rates of PLHIV for different cART eras and age groups were sourced from published literature [10].

For the purposes of this study, we defined a 'class-stage change' as having occurred when a new class of drug is administered to which the patient has not received previously (e.g. if a patient is currently taking N(t)RTI and NNRTI, and has not previously been prescribed a protease inhibitor (PI), then when a PI is prescribed, they are said to be on a new 'classstage'). A class-stage change may occur due to resistance, intolerance or drug toxicity. Exhaustion of cART options occurs when a patient requires a new drug class to ensure successful treatment but no such remaining option for the patient exists.

Clinical practice indicated that only a small percentage (3.2\% in AHOD) of people started HIV therapy with agents selected from three independent drug classes and that it was not common (4.5\%) for two or more classes to be added at a specific regimen change. Hence, in the model we assumed that each individual starts cART with two different classes of antiretroviral drugs and only one new drug class is added per class-stage change. As there are six classes of antiretroviral drug, and most patients start with two different classes, it is assumed that there are a maximum five possible class-stages. 
Based on observed data from our cohort, our simulation model does not include any interclass-stage dependency. Hence, we treated time spent on the second class-stage as independent of the length of time the person spent on their first regimen. Age in the model had no impact on the rate of transition to new class-stages as there is no evidence of age dependence in the rate of progression through treatment options in this cohort [11].

Using data from our cohort we calculated the distribution of time spent in each class-stage with the Kaplan-Meier estimator (KME). Right censoring of data was considered in determining the KME. We then sampled from the KME to obtain times on each regimen for each simulated Individual $(10,000)$. For further details on these calculations see Appendix 1.

Death was simulated in the model based on age-dependent standardised mortality ratios (SMRs) in PLHIV [10]; the SMRs were also calculated for Australian PLHIV. These SMRs were then combined with general population death rates [12] to determine the estimated rate of death of PLHIV. Mortality rates for the general population were used as a control and different mortality rates were used for PLHIV: where exhaustion of cART options does not occur; exhaustion of cART options causes an increase in mortality; and we also considered the scenario of no treatment availability. In the simulations in which PLHIV have a limited number of ART classes available, we assumed that the mortality rate increased to the rate expected in the early cART era (1996-1999) associated with people living with AIDS.

We performed simulations of these scenarios for individuals starting treatment at 20 and 40 years of age. Examination of Australia's surveillance data revealed that $17.2 \%$ of people diagnosed with HIV during 2006-2010 had CD4 counts below 200 at diagnosis. Therefore $17.2 \%$ of people in simulations with treatment available were ascribed death rates of those diagnosed with AIDS in the cART era. It was assumed that the remainder had a death rate equal to that of PLHIV in the cART era who had never been diagnosed with AIDS and were currently using cART. The flow chart in Appendix 2 indicates how the rate of mortality was chosen depending on the circumstances of the individual in the model. Further details of mortality calculations are in Appendix 3.

Sources of uncertainty in our calculations include the stochastic nature of the model and uncertainty in the input parameters. Uncertainty in the parameters are derived from confidence intervals in established rates (such the mortality rate of PLHIV in Nakhaee et al. [10]) and in model assumptions. Specifically, the rate of exit from the fourth and fifth classstage is unknown. We assumed that the fourth and fifth class-stage had a median duration similar to that of the third class-stage but we allowed it to vary between $50-150 \%$ of the third class-stage. Similarly, the rate of mortality of people who have progressed through all available treatments is unknown. We assumed mortality in this group would be $50-150 \%$ of the rate in those with AIDS in the early cART era. Results of uncertainty are reported as the $95 \%$ uncertainty bound [UB], which indicates the range of $95 \%$ of simulation results that are closest to the median.

\section{Results}

The median time on each class-stage varied between class-stages. The median time on the first class-stage was 7.2 years. The second and third class-stages had estimated median durations of 15.2 years and 5.2 years respectively for class-stages that were initiated from 1997 to 2010 . We also investigated limiting the study to class-stages initiated in the latter half of the cART era (years 2004 to 2010) to determine if there was a great impact on treatment duration due to greater tolerability of modern combinations. In the latter half of the cART era, time spent on the first, second and third class-stages were 8.4, 13.6 and 2.5 years respectively. Because there were very limited data on class-stage completion time for 
the years 2004 to 2010 and there was not a great difference between combined class-stage duration over this period compared to 1997-2010, we used all observations to inform our simulations of mortality.

Plots of the Kaplan-Meier estimator of the time on each class-stage initiated from 1997 onwards, and the function estimating the KME, are shown in Fig. 1. The observed treatment data indicates that $10 \%$ of the people who initiate their first class-stage will move on to the second class-stage (i.e. adding a new drug class to which they are naive) within 0.71 (95\% CI $0.61-0.88$ ) years. This compares with the time required for $10 \%$ of people to change from the second-to-third class-stage $(4.2,95 \%$ CI 3.5-5.5 years) and the third-to-fourth class stage class-stage (1.5, 95\% CI 1.0-3.3 years). The second and third class-stages have a slower rates of progression to the next class-stage in the early months of treatment.

The median time until exhaustion of currently available treatment options (without considering mortality) is 45.5 years (IQR $=34.0-61.0$ years). However, approximately $10 \%$ of PLHIV are expected to exhaust all currently available cART options after just 25.6 years, exposing these individuals to higher mortality rates $[13,14]$. In Fig. 2 we present the calculated proportion of people on each of the possible class-stages since treatment initiation.

Using the KME of the treatment cohort data, coupled with the mortality assumptions, we estimated that a male who commences cART at 20 years of age is expected to live to 67.4 (IQR 53.2-77.7) years of age, when adjusting for treatment option exhaustion. This is a substantial improvement on no cART (estimated survival to age 27.7 [IQR 23.8-32.0] years), but is substantially less than the expected life expectancy of a male in the general population (82.2 [IQR 74.0-87.8] years). For those commencing cART at 40 years of age, a smaller gap in life expectancy is predicted (PLHIV 78.4 [IQR 69.7-85.4] years, general population 82.4 [IQR 74.7-87.9] years), as shown in Table 1.

Survival curves of PLHIV at the ages of 20 and 40 years, under the different treatment availability scenarios are shown in Fig. 3. The survival rate of PLHIV is similar but very slightly less than the general population for the first 20 years of treatment. However, a divergence in mortality between PLHIV and the general population appears from around 20 years of treatment experience, as individuals utilise all of their treatment options and hence start having greater mortality rates.

\section{Discussion}

We have demonstrated that while predictions of life expectancy based on observed mortality rates from cohort studies suggest life expectancy approaching, if not reaching, that of the general population, this is dependent upon sequential cART regimens proving to be successful over decades. While our simulated overall median estimate of life expectancy is substantial with the use of all available cART options, our results are clear that a substantial proportion of PLHIV will run out of effective cART options well before they have approached the life expectancy of their HIV-uninfected peers, particularly if HIV infection and cART initiation occurs earlier in life.

While these results confirm the great strides made in improving the life expectancy of PLHIV, they also demonstrate the limitations. This may become even more problematic in the future if the contemporary 'treatment as prevention' strategy becomes mainstream practice, resulting in PLHIV being diagnosed early in the course of their infection and initiating therapy as soon as possible regardless of CD4 level. This strategy has recently been recommended by the International Antiviral Society [15] and is supported by the understanding that treatment is a form of prevention (HPTN 052) and that treatment has 
become safer, less toxic and more likely to be successful [16,17]. What the strategy does not take into account, however, is the inherent limitation of the current HIV armamentarium to support long-term life expectancy. Our results suggest that increases in treatment at high CD4 levels in young individuals may lead to shortfalls in life expectancy if the rate of treatment failure is the same between individuals with high and low CD4 levels. This, in and of itself, is not necessarily disastrous at either an individual or public health level.

Nevertheless, it is an aspect of any early cART intervention initiative that must be taken into account and clearly explained to anyone offered treatment.

An exhaustive search of the most efficient combination of antiretroviral therapies and the order in which they should be administered has not yet been performed. Research to date has mostly focused on the identification of very poor combinations such as combinations that encourage antiretroviral resistance. Current theory for the administration of cART focuses on choosing drugs that have high efficacy and low side effects to administer first, with an aim of minimising cost in the early stages of treatment. By determining the most efficient combination and order of regimen administration, we may be able to extend the viability of currently available cART over a longer period and hence increase the life expectancy of PLHIV by reducing the rate of developing multi-class resistance.

The risk of widespread multiclass resistance and subsequent increase in mortality leads to the conclusion that development of more unique antiretroviral drug classes should be pursued and that the current suite is not yet adequate. Recently, the field has seen how hard it has become to get a new drug to market even in an existing class. The late stage clinical trials of the second in class CCR5-antagonist Vicriviroc did not meet their primary efficacy endpoints [18] and consequently the originator company decided in 2010 to not pursue regulatory approval for the drug [19].

While the field is currently reinvigorated by notions of cure [20], this would seem to be at least many decades away and may never be possible. Similarly, while excitement about the controversial results of the Thai vaccine is opening up potentially fruitful areas of investigation, we seem still to be very far from developing an effective vaccine [21,22]. Probably the most immediate prevention strategy that may see implementation is the use of pre-exposure prophylaxis and the use of early initiation of treatment [23]. The penalty of such an approach at a population-level must inevitably be the selection and transmission of drug resistance which will once again impinge upon the projected life expectancy of any individual so infected. Transmitted drug resistance (TDR) was not considered when determining the time spent on each class-stage in our model. If TDR were to become highly prevalent within the population of PLHIV, the time spent on each class-stage would likely be reduced. This may result in a reduction in the total time each cART user experiences on therapy, and could potentially increase mortality among PLHIV.

The study has a number of limitations. Data used in this study were sourced from Australia and hence these results may not be applicable in all settings. That said, the Australian HIV epidemic reflects aspects of the HIV epidemic amongst MSM in the developed world. The Australian data used for this study contains relatively little information about female PLHIV and relatively few patients with viral hepatitis. We therefore did not include the results in the manuscript for females living with HIV. The AHOD cohort is also a heavily treated population, receiving greater levels of care than would be expected in the wider population of PLHIV, meaning the results are representative of the treated population, but not necessarily of the broader population of PLHIV. The AHOD cohort was also primarily recruited in the early years of cART. This means that they have been more exposed to treatments that have higher toxicity and greater rates of resistance development than those 
that a person receiving modern standards of care would receive. The implication of this is that the model may underestimate the time spent on first and second class-stages.

There are limitations in the simulation of older age groups. Specifically Nakhaee et al. [10] describe the SMRs of PLHIV by age group, including those aged 65 years and older. It is unlikely that these SMRs will be accurate for ages much beyond the age of 80 years. As our study used all-cause mortality rates, there may also be limitations in predicting mortality due to changing rates of comorbid conditions and their treatment.

For people diagnosed with HIV later in life, currently available treatments appear to be sufficient to provide lifelong treatment options. However, people infected at a younger age will be more likely to outlive their treatment options. Research into antiretroviral drugs for the treatment of HIV has had massive investment and a number of different drugs of several class types were rapidly developed. That said, this pace of development is unlikely to continue in the future.

An era of treatment exhaustion is approaching. People who initiated cART in 1997 have already had 15 years of treatment experience by the year 2012. We estimate that in 10 years time, $10 \%$ of PLHIV who began treatment with triple therapy and using two distinct drug classes in 1997 have no more treatment options available to them. It should be noted that initiating triple therapy is our 'best case scenario': there are many who started mono or dual therapy prior to 1997 who may have developed class resistance earlier than those on triple therapy. These people will likely exhaust their treatment options earlier than those who started with an effective triple therapy.

Complacency about the adequacy of existing treatments in affording PLHIV full life expectancy needs to be reconsidered. The development of more drugs, specifically drugs available in new drug classes with mechanisms of antiretroviral action independent of the current available range, is necessary to support a near-normal life expectancy for the majority of PLHIV.

\section{Acknowledgments}

This study was funded from the following sources: the Australian Government Department of Health and Ageing; Australian Research Council (DP1093026; FT0991990). The views expressed in this publication do not necessarily represent the position of the Australian Government. The Australian HIV Observational Database is funded as part of the Asia Pacific HIV Observational Database, a program of The Foundation for AIDS Research, amfAR, and is supported in part by a grant from the U.S. National Institutes of Health's National Institute of Allergy and Infectious Diseases (NIAID) (Grant No. U01-AI069907) and by unconditional grants from Merck Sharp \& Dohme; Gilead; Bristol-Myers Squibb; Boehringer Ingelheim; Roche; Pfizer; GlaxoSmithKline; Janssen-Cilag.

\section{References}

1. Lohse N, Hansen A-BE, Pedersen G, Kronborg G, Gerstoft J, Sørensen HT, et al. Survival of Persons with and without HIV Infection in Denmark, 1995-2005. Annals of Internal Medicine. 2007; 146:87-95. [PubMed: 17227932]

2. The Antiretroviral Therapy Cohort Collaboration. Life expectancy of individuals on combination antiretroviral therapy in high-income countries: a collaborative analysis of 14 cohort studies. The Lancet. 372:293-299.

3. Bhaskaran K, Hamouda O, Sannes M, Boufassa F, Johnson AM, Lambert PC, et al. Changes in the Risk of Death After HIV Seroconversion Compared With Mortality in the General Population. JAMA: The Journal of the American Medical Association. 2008; 300:51-59. [PubMed: 18594040]

4. van Sighem A, Gras L, Reiss P, Brinkman K, de Wolf F. study obotAnoc. Life expectancy of recently diagnosed asymptomatic $\mathrm{HIV}$-infected patients approaches that of uninfected individuals. AIDS. 2010; 24:1527-1535. 1510.1097/QAD.1520b1013e32833a33946. [PubMed: 20467289] 
5. Hogg, R.; Samji, H.; Cescon, A.; Modur, S.; Napravnik, S.; Martin, J., et al. Temporal Changes in Life Expectancy of HIV+ Individuals: North America. Conference on Retroviruses and Opportunistic Infections; Seattle. 2012.

6. Gulick R. New antiretrovirals. Retrovirology. 2012; 9:I7.

7. The Strategies for Management of Antiretroviral Therapy Study Group. Inferior Clinical Outcome of the CD4+ Cell Count-Guided Antiretroviral Treatment Interruption Strategy in the SMART Study: Role of CD4+ Cell Counts and HIV RNA Levels during Follow-up. Journal of Infectious Diseases. 2008; 197:1145-1155. [PubMed: 18476293]

8. Cohen MS, Chen YQ, McCauley M, Gamble T, Hosseinipour MC, Kumarasamy N, et al. Prevention of HIV-1 Infection with Early Antiretroviral Therapy. New England Journal of Medicine. 2011; 365:493-505. [PubMed: 21767103]

9. The Australian H. I. V Observational Database. Rates of combination antiretroviral treatment change in Australia, 1997-2000. HIV Medicine. 2002; 3:28-36. [PubMed: 12059948]

10. Nakhaee F, Black D, Wand H, McDonald A, Law M. Changes in mortality following HIV and AIDS and estimation of the number of people living with diagnosed HIV/AIDS in Australia, 1981-2003. Sexual Health. 2009; 6:129-134. [PubMed: 19457292]

11. Pour SM, Woolley I, Canavan P, Chuah J, Russell DB, Law M, et al. Triple class experience after initiation of combination antiretroviral treatment in Australia: survival and projections. Sexual Health. 2011; 8:295-303. [PubMed: 21851768]

12. Australian Bureau of Statistics (ABS). Statistical Geography Volume 1 - Australian Standard Geographical Classification (ASGC). Canberra: ABS; 2006.

13. Falster K, Choi JY, Donovan B, Duncombe C, Mulhall B, Sowden D, et al. AIDS-related and nonAIDS-related mortality in the Asia-Pacific region in the era of combination antiretroviral treatment. AIDS. 2009; 23:2323-2336. 2310.1097/QAD.2320b2013e328331910c. [PubMed: 19752715]

14. McManus, H.; O’Connor, CC.; Boyd, M.; Broom, J.; Russell, D.; Watson, K., et al. Examination of long term survival in HIV positive patients with up to 15 years of antiretroviral therapy. Australasian HIV/AIDS Conference; Canberra. 2011.

15. Thompson, MaAJAHJF., et al. Antiretroviral treatment of adult hiv infection: 2012 recommendations of the international antiviral society-usa panel. JAMA: The Journal of the American Medical Association. 2012; 308:387-402. [PubMed: 22820792]

16. Bartlett JA, Fath MJ, DeMasi R, Hermes A, Quinn J, Mondou E, et al. An updated systematic overview of triple combination therapy in antiretroviral-naive HIV-infected adults. AIDS. 2006; 20:2051-2064. 2010.1097/2001.aids.0000247578.0000208449.ff. [PubMed: 17053351]

17. Boyd MA. Improvements in antiretroviral therapy outcomes over calendar time. Current Opinion in HIV and AIDS. 2009; 4:194-199. 110.1097/COH.1090b1013e328329fc328328d. [PubMed: 19532050]

18. Gathe, J.; Diaz, R.; Fatkenheuer, G.; Zeinecker, J.; Mak, C.; Vilchez, R., et al. Phase 3 Trials of Vicriviroc in Treatment-experienced Subjects Demonstrate Safety but Not Significantly Superior Efficacy over Potent Background Regimens Alone (\# 54LB). 17th Conference on Retroviruses and Opportunistic Infections; San Francisco. 2010;

19. Loftus, P. The Wall Street Journal. New York: 2010. Merck Won't Seek FDA Approval For HIV Drug.

20. Lafeuillade A. Potential Strategies for an HIV Infection Cure. HIV Clin Trials. 2001; 12:121-130. [PubMed: 21684852]

21. de Souza MS, Ratto-Kim S, Chuenarom W, Schuetz A, Chanta-kulkij S, Nuntapinit B, et al. The Thai Phase III Trial (RV144) Vaccine Regimen Induces T Cell Responses That Preferentially Target Epitopes within the V2 Region of HIV-1 Envelope. The Journal of Immunology. 2012

22. Montefiori DC, Karnasuta C, Huang Y, Ahmed H, Gilbert P, de Souza MS, et al. Magnitude and Breadth of the Neutralizing Antibody Response in the RV144 and Vax003 HIV-1 Vaccine Efficacy Trials. Journal of Infectious Diseases. 2012

23. Celum C. HIV preexposure prophylaxis: new data and potential use. Top Antivir Med. 2011; 19:181-185. [PubMed: 22298887] 
24. Mellors JW, Munoz A, Giorgi JV, Margolick JB, Tassoni CJ, Gupta P, et al. Plasma Viral Load and CD4+ Lymphocytes as Prognostic Markers of HIV-1 Infection. Annals of Internal Medicine. 1997; 126:946-954. [PubMed: 9182471] 

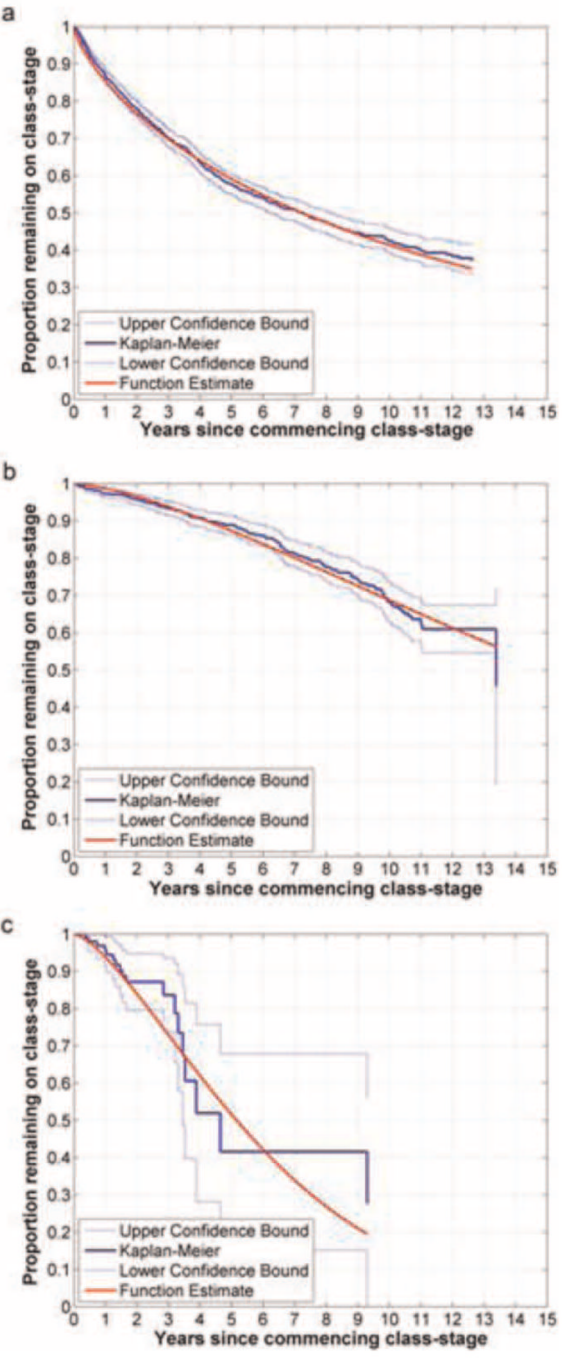

Fig. 1.

Kaplan-Meier estimator of the duration of the first 4 class-stages. (a) Class-stage 1 (initial therapy of 2 different class stages, until addition of a class of ART to which the patient is naive); (b) Class-stage 2 (time spent as a 3 ART class experienced patient); (c) Class-stage 3 (time spent as a 4 ART class experienced patient). 


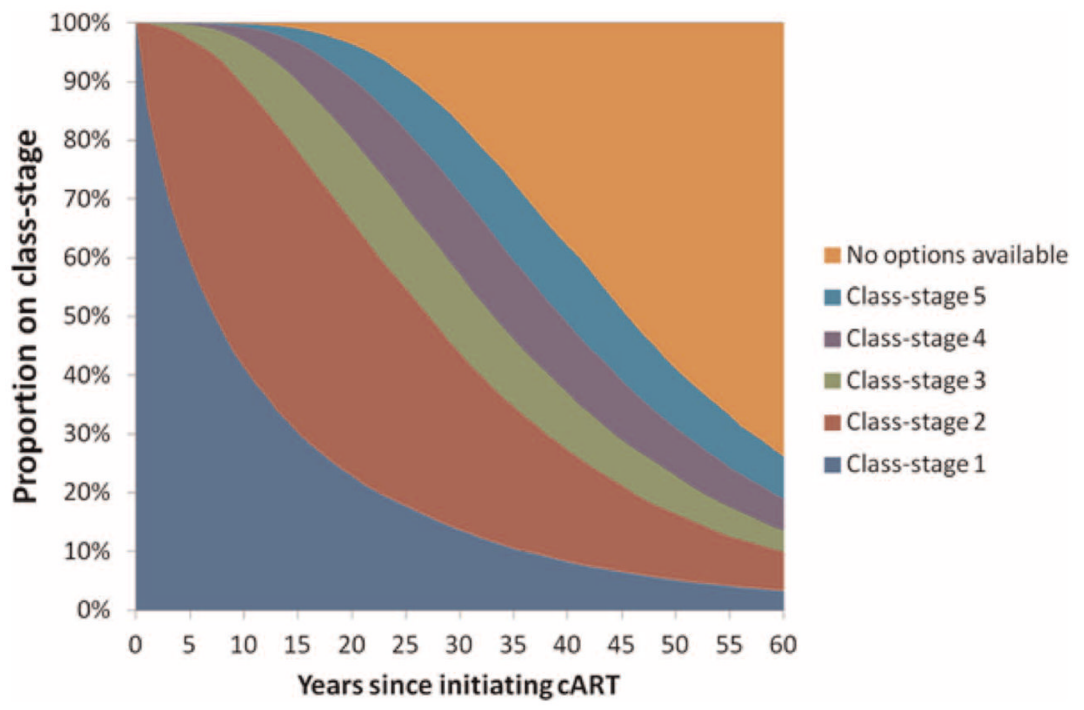

Fig. 2.

Proportion of people on each of the class-stages in a scenario in which 5 class-stages are available. The upper orange region indicates individuals who have exhausted all their cART options. 

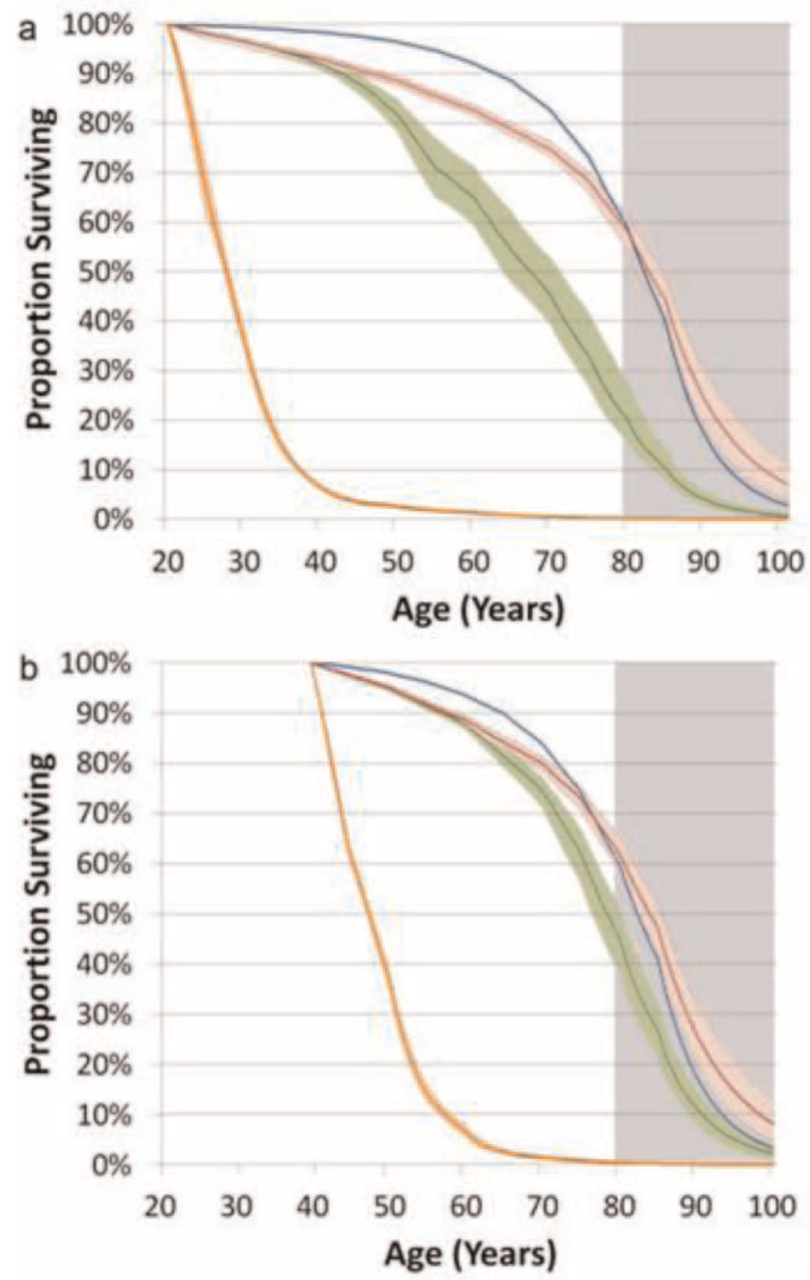

Fig. 3.

The proportion of men surviving by age when diagnosed at age 20 (a), and age 40 (b). The scenarios represented include: the male general population in Australia (blue); ii) PLHIV, with unlimited class-stages available (red); iii) PLHIV, 5 class-stages available (number of class-stages currently available in 2012) (green); iv) PLHIV, no cART available (orange). The heavy lines indicate the median of the scenarios under the varying parameter values. The colour shaded areas indicate the range of the median $95 \%$ of simulations which results from the varying parameter values and stochasticity of the model. The greyed area indicates the region in which we are uncertain of the suitability of mortality rates in the older population of PLHIV. 


\section{Table 1}

Median age (and inter-quartile range) at death for men with diagnosed HIV under various treatment scenarios. The unlimited drug availability scenario is a theoretical scenario to simulate life expectancy if individuals never face the prospect of having no new treatments to act as replacements for exhausted treatments that are currently available.

\begin{tabular}{|c|c|c|c|}
\hline \multirow[t]{2}{*}{ Population } & \multirow{2}{*}{$\begin{array}{l}\text { Number of ART classes } \\
\text { available }\end{array}$} & \multicolumn{2}{|l|}{ Median age of mortality (IQR) } \\
\hline & & $\begin{array}{l}\text { Initiate treatment/observation } \\
\text { at age } 20 \text { years }\end{array}$ & $\begin{array}{l}\text { Initiate treatment/observation } \\
\text { at age } 40 \text { years }\end{array}$ \\
\hline \multirow[t]{3}{*}{ HIV infected } & None & $27.7(23.8-32.0)$ & $47.5(43.3-52.1)$ \\
\hline & 6 (current scenario) & $67.4(53.2-77.7)$ & $78.4(69.7-85.4)$ \\
\hline & Unlimited & $82.8(69.6-89.9)$ & $84.0(73.6-90.4)$ \\
\hline General population (non-HIV) & $N A$ & $82.2(74.0-87.8)$ & $82.4(74.7-87.9)$ \\
\hline
\end{tabular}

